$$
\begin{aligned}
z(x, y) & =\int_{0}^{x} d x_{1} \int_{0}^{y} z_{x y}\left(x_{1}, y_{1}\right) d y_{1}+\int_{0}^{y} d y_{1} \int_{0}^{y_{1}} z_{y y}\left(0, y_{2}\right) d y_{2} \\
+ & \int_{0}^{x} d x_{1} \int_{0}^{x_{1}} z_{x x}\left(x_{2}, 0\right) d x_{2}+z_{y}(0,0) y+z_{x}(0,0) x+z(0,0) .
\end{aligned}
$$

Proceeding as before,

$$
\begin{aligned}
U[z(x, y)]=V\left[z_{x y}(x, y)\right]+V_{1}\left[z_{y y}(0, y)\right]+V_{2}\left[z_{x x}(x, 0)\right] \\
\\
+z_{y}(0,0) U[y]+z_{x}(0,0) U[x]+z(0,0) U[1],
\end{aligned}
$$

where $V, V_{1}$ and $V_{2}$ are linear and continuous of the zeroth order and $U[y], U[x]$ and $U[1]$ are constants.

Columbia University, June, 1916 .

\title{
THE EQUATION OF A PLANE RATIONAL CURVE DEFINED BY PARAMETRIC EQUATIONS.
}

\author{
BY HERBERT WILLIAM RICHMOND.
}

The explicit equation $F(x, y, z)=0$ of a rational plane curve, defined by the parametric equations

$$
x: y: z=A(t): B(t): C(t)
$$

where $A(t), B(t), C(t)$ are polynomials of order $n$ in $t$, is expressed by Salmon (Higher Plane Curves, $\S 44$ ) as a determinant of $3 n$ rows. In the April number of the Bulletin Professor J. E. Rowe exhibits this equation as a symmetrical determinant of $n$ rows, in which each element is a linear function of $x, y, z$. It has been my custom when lecturing upon algebraic geometry to obtain this form of equation as follows:

Let $(x, y, z)$ be the coordinates of the point whose parameter is $t$, and let $s$ be any value of the parameter; then

$$
\left|\begin{array}{lll}
x & A(t) & A(s) \\
z & C(t) & C(s) \\
y & B(t) & B(s)
\end{array}\right|(=\Delta \mathrm{say})
$$

vanishes for every value of $s$. Imagine the determinant expanded and the factor $s-t$ removed. We have now a 
polynomial in $s$ of order $n-1$, which vanishes for all values of $s$; the $n$ coefficients of powers of $s$ severally vanish. But each coefficient is a polynomial in $t$ of order $n-1$, every power of $t$ being multiplied by a linear function of $x, y, z$. These powers of $t$ may be eliminated by a determinant of $n$ rows, which is the equation of the curve.

The linear function of $(x, y, z)$ which in the $r$ th row and $s$ th column is the coefficient of $s^{n-r} t^{n-s}$ in the expanded form of $\Delta /(s-t)$, and is readily written down if need be. Just as Salmon's method is an adaptation of Euler's (or Sylvester's) method of eliminating a variable from two polynomials, so the foregoing is an adaptation of Cayley's statement of Bezout's method.

King's College,

Cambridge, England.

\section{SCIENTIFIC METHOD IN PHILOSOPHY.}

Our Knowledge of the External World as a Field for Scientific Method in Philosophy. By Bertrand Russeld, M.A., F.R.S., Late Fellow of Trinity College, Cambridge. Chicago, London, The Open Court Publishing Company, 1914. vii $+245 \mathrm{pp}$.

THE philosophic method in science is a thing familiar enough, in sound if not in fact. But what, pray, can be meant by "scientific method in philosophy"? That is what Mr. Russell essays to make clear, in outline at all events, in this book, and he does it partly by a general critique on the methodologies of philosophy and science and partly, indeed mainly, by handling certain specific problems of philosophy in the spirit and the manner of science. The chief ones among these illustrative examples belong to the general problem of the relation between the data of sense and the time, space, and matter of mathematical physics. The discussion is guided by a highly important and exceedingly difficult aim. The aim is to sketch a method available in all departments of philosophy and "adequate to yield whatever objective scientific knowledge it is possible to obtain." Traditional philosophy, however worthy of studious attention, has been too impatient; it has been too ambitious; its pretensions and claims have been grossly extravagant; it has not 